\title{
Modeling of Mechanical Properties of Concrete Mixed with Expansive Additive
}

\author{
Hyeonggil Choi ${ }^{1)}$, and Takafumi Noguchi ${ }^{2), *}$
}

(Received December 8, 2014, Accepted August 23, 2015, Published online September 16, 2015)

\begin{abstract}
This study modeled the compressive strength and elastic modulus of hardened cement that had been treated with an expansive additive to reduce shrinkage, in order to determine the mechanical properties of the material. In hardened cement paste with an expansive additive, hydrates are generated as a result of the hydration between the cement and expansive additive. These hydrates then fill up the pores in the hardened cement. Consequently, a dense, compact structure is formed through the contact between the particles of the expansive additive and the cement, which leads to the manifestation of the strength and elastic modulus. Hence, in this study, the compressive strength and elastic modulus were modeled based on the concept of the mutual contact area of the particles, taking into consideration the extent of the cohesion between particles and the structure formation by the particles. The compressive strength of the material was modeled by considering the relationship between the porosity and the distributional probability of the weakest points, i.e., points that could lead to fracture, in the continuum. The approach used for modeling the elastic modulus considered the pore structure between the particles, which are responsible for transmitting the tensile force, along with the state of compaction of the hydration products, as described by the coefficient of the effective radius. The results of an experimental verification of the model showed that the values predicted by the model correlated closely with the experimental values.
\end{abstract}

Keywords: expansive additive, compressive strength, elastic modulus, pore volume, modeling.

\section{Introduction}

The application of an expansive additive is known to be an effective means of reducing shrinkage and increasing crack resistance. Thus, such an application is gradually becoming more common in construction projects (Choi et al. 2012a, b). In this study, we attempted to theoretically model the compressive strength and elastic modulus of hardened cement that had been treated with an expansive additive to reduce the shrinkage. The compressive strength and elastic modulus of hardened cement paste mixed with an expansive additive are closely related to the structure formation of the hardened cement paste by the hydration reaction of the cement and expansive additive (AlRawi 1976; Woods 1933; Taplin 1959). In other words, hydrates are generated as a result of the hydration between the cement and expansive additive; these hydrates then fill up the pores in the hardened cement. Consequently, a dense, compact structure is formed through the contact between the particles of the expansive additive and cement, which leads to the

\footnotetext{
${ }^{1)}$ Graduate School of Engineering, Muroran Institute of Technology, Hokkaido, Japan.

${ }^{2)}$ Graduate School of Engineering, The University of Tokyo, Tokyo, Japan.

*Corresponding Author; E-mail: noguchi@bme.arch. t.u-tokyo.ac.jp

Copyright ( $\odot$ The Author(s) 2015. This article is published with open access at Springerlink.com
}

manifestation of the strength and elastic modulus. Hence, it is important to determine the change in the organizational structure of a hardened cement paste by the progression of the hydration to estimate the strength and elastic modulus of a hardened cement paste mixed with an expansive additive.

In this study, the compressive strength and elastic modulus of a hardened cement paste mixed with an expansive additive were modeled and validated by considering the pore structure of a hardened cement paste based on the hydration, as shown in Fig. 1.

\section{Modeling of Compressive Strength}

\subsection{Equation of Strength Development}

Many studies have reported the relationship between the pore volume and strength (Maruyama 2003; Ryshkewitch 1953; Schiller 1958). In this study, it was assumed that the strength development of a cement paste mixed with an expansive additive was closely related to the pore volume based on the results of the existing studies. A typical example showing that the strength development behavior demonstrates an application possibility from the viewpoint of the pore volume and strength of a cement paste is shown in the following equations of Ryshkewitch (Ryshkewitch 1953) and Schiller (Schiller 1958).

$$
\begin{aligned}
f_{c}= & f_{o} \cdot e^{-B P} \quad \text { (Ryshkewitch's equation) } \\
& : \text { In the case of low porosity }
\end{aligned}
$$




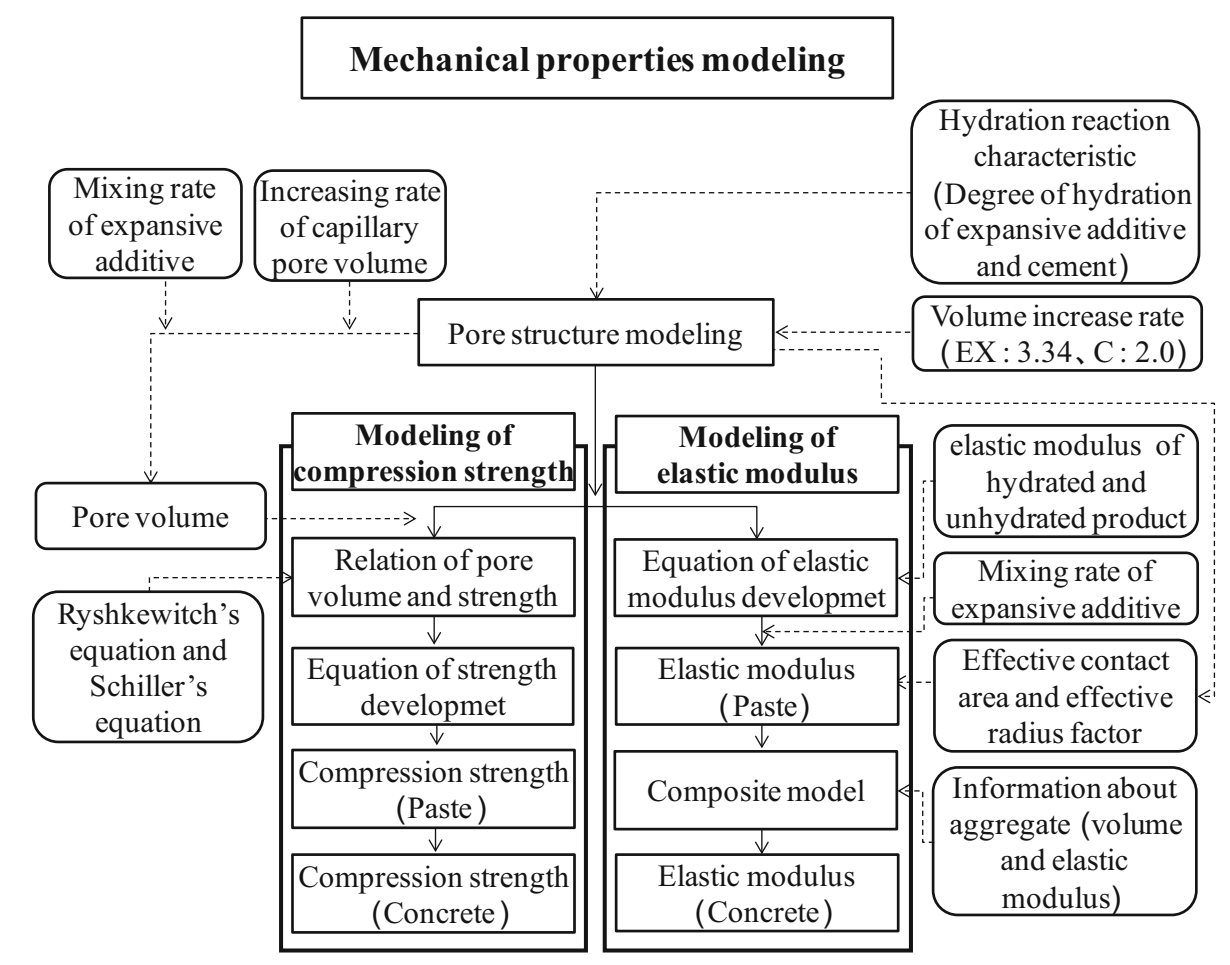

Fig. 1 Flow of mechanical properties modeling of concrete using expansive additive.

$f_{c}=C \cdot \ln \left(P_{c r} / P\right)$

(Schiller's equation): In the case of high porosity

where $f_{c}$ is the compressive strength of a hardened cement paste, $P$ is the porosity, $f_{o}$ is the strength of the hardened cement paste when the porosity is zero, $P_{c r}$ is the porosity of the hardened cement paste when the strength is zero and $B$ and $C$ are material constants.

Similar to the above equations of Ryshkewitch (1953) and Schiller (1958), many researchers have suggested various forms for the equations describing the relationship between the porosity and strength. However, the relationships represented by these equations are rather simple, and their applicability is limited because only experimental values were used for the parameters in the equations. On the other hand, because all of the cement particles are the same size, Katsura et al. (1996) defined the strength of a cube of cement paste as the cement's original strength, where the strength of the hydrated cement paste could be expressed in terms of the contact between the cement particles within the cube produced by hydration. This strength is the strength of a cement paste with zero porosity. That is, the relative strength $R_{S}=f_{0} / f$, which is the ratio of the original strength $f_{o}$ to the strength with pores $f$, is compared to the contact area, which has a linear relationship with the porosity, suggesting that the $B$ value of Ryshkewitch's equation is 5.0, and the $P_{c r}$ value of Schiller's equation is 0.523 . In addition, in the C-CBM model suggested by Maruyama (2003), based on the assumption of this contact area, the contact area $A_{c}$ produced by increasing the maximum radius of a hydrate $R_{t}$ is modeled and applied in the following equation.

$$
\begin{aligned}
& \text { Case of }\left(0.5 \leq R_{t}<\sqrt{2} / 2\right): \\
& A_{c}=\pi\left(R_{t}^{2}-0.5^{2}\right) \\
& \text { Cas of }\left(\sqrt{2} / 2 \leq R_{t}<\sqrt{3} / 2\right) \\
& A_{c}=8\left\{\frac{1}{2}\left(R_{t}^{2}-0.5^{2}\right)\left(\frac{\pi}{4}-A \operatorname{COS}\left(\frac{0.5}{\sqrt{R_{t}^{2}-0.5^{2}}}\right)\right)\right. \\
& \left.+0.25 \sqrt{R_{t}^{2}-0.5}\right\}
\end{aligned}
$$

The suggested $B$ value of Ryshkewitch's equation is 5.43 for a low pore volume of less than 0.3 based on the concept of the contact area in the C-CBM model (Maruyama 2003). In addition, for the high-porosity range, values of 64.5 and 0.523 are suggested for $C$ and $P_{c r}$ of Schiller's equation, respectively, based on the least squares method, with the data showing the relationship between the porosity and strength from Schiller's equation.

In this study, based on the equations of Ryshkewitch (Ryshkewitch 1953) and Schiller (1958), we referred to the existing studies of Maruyama (2003; Katsura et al. 1996) to calculate the coefficient of each porosity. We suggest the following equations for the compressive strength based on the relationship between the strength and porosity.

$$
\begin{aligned}
& f_{c}=182 \cdot e^{-5.215 P}: 0.3>P \\
& f_{c}=73 \cdot \ln (0.523 / P): 0.3 \leq P
\end{aligned}
$$

\subsection{Modeling of Micro-pore Structure}

The total porosity of cement paste mixed with an expansive additive was acquired by adding the porosities of the 
cement and expansive additive parts based on a hydration reaction. This study utilized an existing space formation model (Maruyama 2003; Park and Lee 2005; Park 2004) based on the hydration of the cement, which was used to acquire the porosities of the cement and expansive additive.

As shown in Fig. 2, the entire modeling area is represented as a $1 \mathrm{~cm}^{3}$ cube. The density of water is denoted as $\rho_{w}$, the density of the expansive additives is denoted as $\rho_{e}$, and the water-to-binder ratio is denoted as $x$. Given these conditions, the volume of the expansive additive in the cube can be expressed as follows:

$$
V_{c, e}=\frac{1}{x \cdot \frac{\rho_{c, e}}{\rho_{w}}+1}
$$

The radius $r_{o}$ of a particle of the expansive additives in the $1 \mathrm{~cm}^{3}$ cube is obtained as

$$
r_{o}=\left(\frac{3 V_{c, e}}{4 \pi}\right)^{\frac{1}{3}}
$$

If the particle radii of the expansive additive before and after the hydration reaction are assumed to be $r_{o}$ and $r_{u}$, respectively, and if the outermost radius of the particle after the reaction is assumed to be $R_{t}$, then the hydration reaction rate can be defined as the volume of the hydration reaction divided by the volume of the expansive additive before the hydration reaction. Thus, the following equation is obtained:

$$
\alpha=\frac{\frac{4}{3} \pi r_{o}^{3}-\frac{4}{3} \pi r_{u}^{3}}{\frac{4}{3} \pi r_{o}^{3}}=1-\left(\frac{r_{u}}{r_{o}}\right)^{3}
$$

In addition, the volume increase rate $V$ can be defined as the volume of the produced hydration product divided by the volume of the portion of the expansive additive that has undergone the reaction. Thus, $V$ can be expressed as

$$
V=\frac{\frac{4}{3} \pi R_{t}^{3}-\frac{4}{3} \pi r_{u}^{3}}{\frac{4}{3} \pi r_{o}^{3}-\frac{4}{3} \pi r_{u}^{3}}=\frac{R_{t}^{3}-r_{u}^{3}}{r_{o}^{3}-r_{u}^{3}}
$$

From Eqs. (8) and (9), the following equation can be obtained:

$$
R_{t}=(1+(V-1) \alpha)^{\frac{1}{3}} \cdot r_{o}
$$

Here, the hydration reaction rate $a$ can be used to define the amounts of cement and expansive additive that have
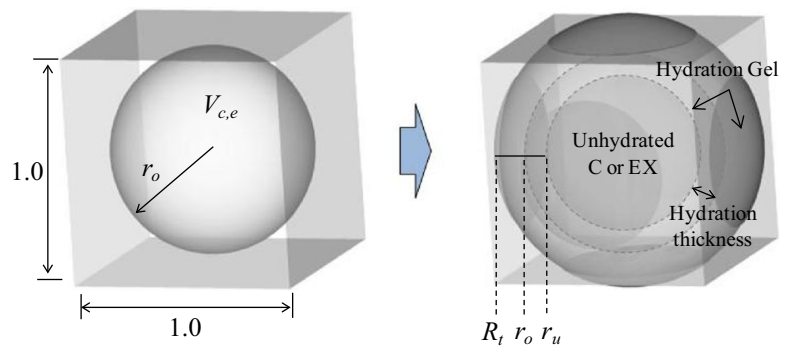

Fig. 2 Hydration reaction of cement and expansive additive particles. reacted from their corresponding total amounts. The calories generated during hydration were measured using a multimicrocalorimeter (MMC-511 SV), and this value was used to determine the hydration reaction rate.

When both the W/C and water/expansive additive ratios are 0.50 , the net calorific values are $437.53 \mathrm{~J} / \mathrm{g}$ and $887.12 \mathrm{~J} /$ $\mathrm{g}$ for the cement and expansive additive, respectively. Figure 3 shows an example of the calculations for the rate of heat liberation and degree of hydration. These confirmed that at every age, the hydration reaction rate for the expansive additive was higher than that of the cement, because of an increase in the exothermic peak through the rapid reaction of the expansive additive at an early age.

On the other hand, for the cement volume that contributes to the reaction in the hydration reaction model, the volume increase rate $V$ of the cement is obtained by adding approximately $75 \%$ of the water volume in the total volume of gel with the volume of gel produced through the hydration reaction by chemically combining cement and water that constitute approximately $25 \%$ of the weight of cement (Tashiro 1993). Therefore, the volume changes calculated using the cement density indicate that the appropriate volume increase rate during the complete hydration of the cement is approximately 1.59 . However, the volume increase rate is expected to increase if the adsorbed water present in the hydration product or the water in the gel pores that does not contribute to the hydration is considered. For this reason, in previous studies (Powers and Brownyard 1947; van Breugel 1997), the volume increase rate of cement was defined as being in the range of 1.9-2.2. Using this range as a reference, in this study, we set the volume increase rate to 2.0 by considering the time that it converged to a certain value with regard to the hydration reaction rate, along with the adsorbed water present in the hydration product or the water in the gel pores that did not contribute to the hydration reaction.

In previous studies (Yanimoto et al. 2003) that described the expansion mechanism of an expansive additive, the volume increase rate $V$ of the expansive additive was acquired with the assumption that the hydration products, which expand due to the expansive additive, were calcium hydroxide and ettringite. Yaniamoto et al. used the volume

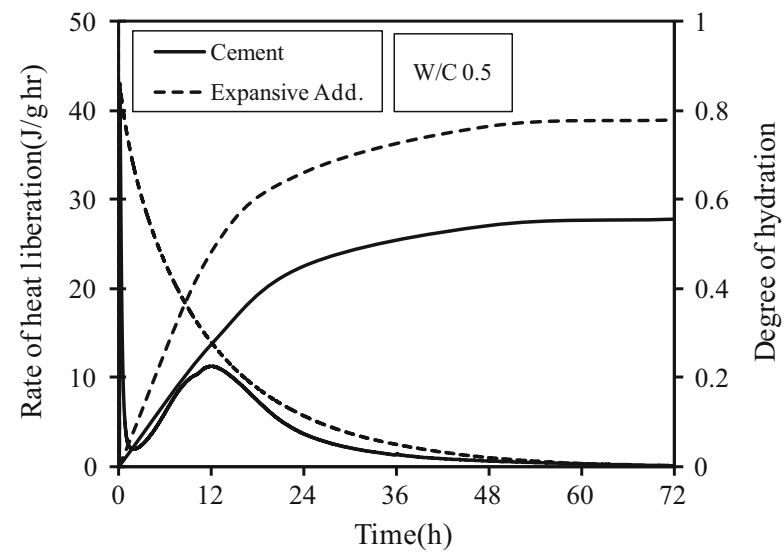

Fig. 3 Rate of heat liberation and degree of hydration. 
of the layer of a hydrate product containing pores that was generated by the expansive additive for the volume of the hydration product that contributed to the expansion by the expansive additive. Here, in relation to the assumption made by Yaniamoto et al. about their model, we note that for a hydration product produced by hydration, a mixed layer containing pores of calcium hydroxide and ettringite is formed on the particle surface of the expansive additive. In addition, half of the ettringite generated by the expansive additive occurs on the surface of the $\mathrm{C}_{3} \mathrm{~A}$ in the cement. In other words, in a case where only the reaction of the expansive additive is considered, it is necessary to consider the ettringite hydration product on the surface of the $\mathrm{C}_{3} \mathrm{~A}$ in the cement. Therefore, in this study, the volume of the hydration product layer produced by the hydration of only the expansive additive was acquired by considering the volume ratio of each of the assumed hydration products (calcium hydroxide and ettringite) that were expanded as a result of the expansive additive. Figure 4 shows the volume of the ettringite hydration product created on the surface of the $\mathrm{C}_{3} \mathrm{~A}$ in the cement by the hydration of the expansive additive. This is the volume of the hydration product with the porosity created by the hydration reaction of the expansive additive. Considering the volume change of the hydrates, as previously discussed, the volume increase rate $V$ of the expansive additive is defined as being 3.34 , which is a relatively constant value.

The outermost radius $R_{t}$ of the cement and expansive additive particles can be acquired using Eq. 10 by considering the volume increase rate $V$. Figure 5 shows a schematic view of the space formation of the cement and expansive additive particles by the outermost radius $R_{t}$. The cement particles are divided into an unlimited period, an early period of contact, and a late period of contact based on the outermost radius $R_{t}$. Then, based on the surface area and volume of each particle, the porosity can be calculated by subtracting the volumes of the particles from the volume of the cube.

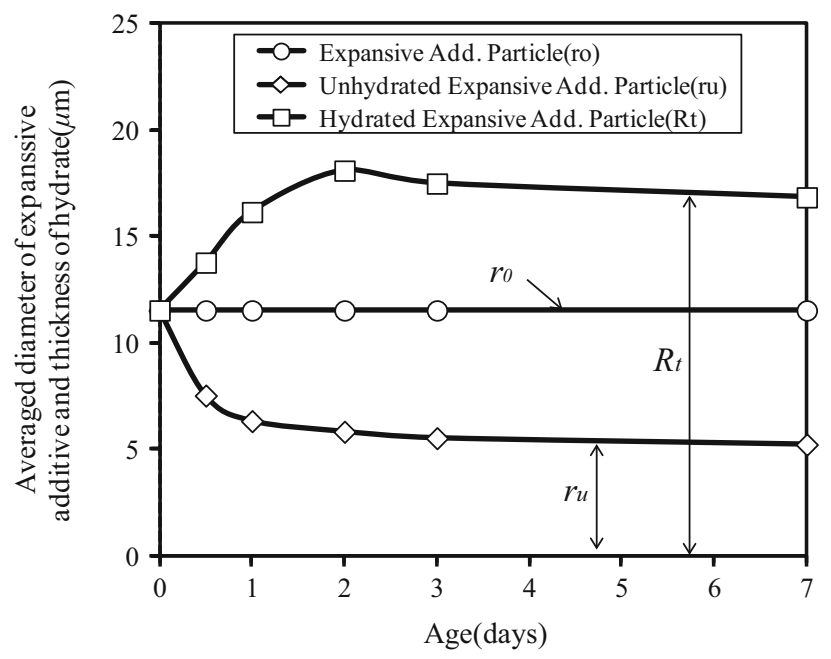

Fig. 4 Volume change of hydration products with expansive additive.
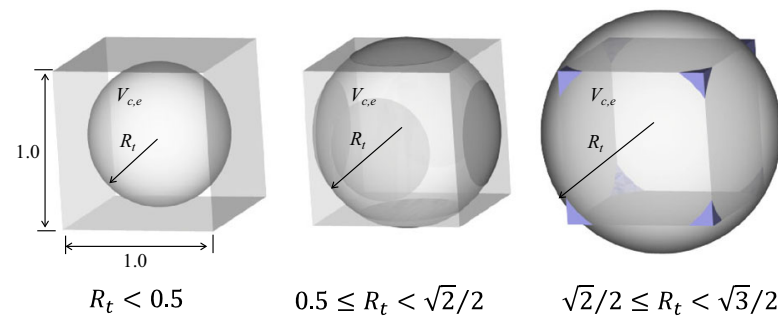

$$
R_{t}<0.5
$$

$0.5 \leq R_{t}<\sqrt{2} / 2$

$\sqrt{2} / 2 \leq R_{t}<\sqrt{3} / 2$

Fig. 5 Schematic view of space formation of cement and expansive additive particles (Maruyama 2003; Park 2004).

On the other hands, with the addition of an expansive additive, the hydration reaction generates capillary pores with a size range of 0.1 to several micrometers, which cause expansion. The volume increase rate of the expansive additive $V$ already contains the pores created by the hydration reaction. Therefore, those pores need to be considered. In other words, the porosity of the expansive additive can be acquired from the values listed in Table 1, which consider the incremental factor $p_{E X}$ of the capillary pores by the volume expansion of the expansive additive. Incremental factor $p_{E X}$ of the capillary pores was acquired by deducing a regression equation for the porosity increase rate according to the mixing rate of the expansive additive by referring to the experimental results of existing studies (Yanimoto et al. 2003; Morioka et al. 1999).

Therefore, the total pore volume of the cement paste mixed with the expansive additive can be expressed by the following equation, depending on the mixture rate of the expansive additive according to the pore volume of the cement and expansive additive required by the hydration reaction

$$
P_{c+E X}=P_{c} \cdot C_{V(\%)}+P_{E X} \cdot E X_{V(\%)}
$$

where $P_{c+E X}$ is the porosity of the cement paste mixed with the expansive additive, $P_{c}$ is the porosity of the cement part, $P_{E X}$ is the porosity of the expansive additive part, $C_{V(\%)}$ is the volume mixing rate of the cement, and $E X_{V(\%)}$ is the volume mixing rate of the expansive additive.

The previous discussion showed that the compressive strength of cement paste mixed with an expansive additive can be acquired using Eqs. (4) and (5), which show the relations of the pore volume and strength at each range of porosity using the total pore volume acquired by Eq. (11).

\section{Modeling of Elastic Modulus}

\subsection{Elastic Modulus of Cement Paste Without Pore}

For the strength development of hardened cement, the strength and porosity seem to be highly correlated because of the stress concentration, which occurs as a result of the strength and pores of the hydration product. However, it is not easy to determine the characteristics of the elastic modulus using only its relationship with the pores because 
Table 1 Area of surface, and volume and porosity of particles by outermost radius $R_{t}$ (Maruyama 2003).

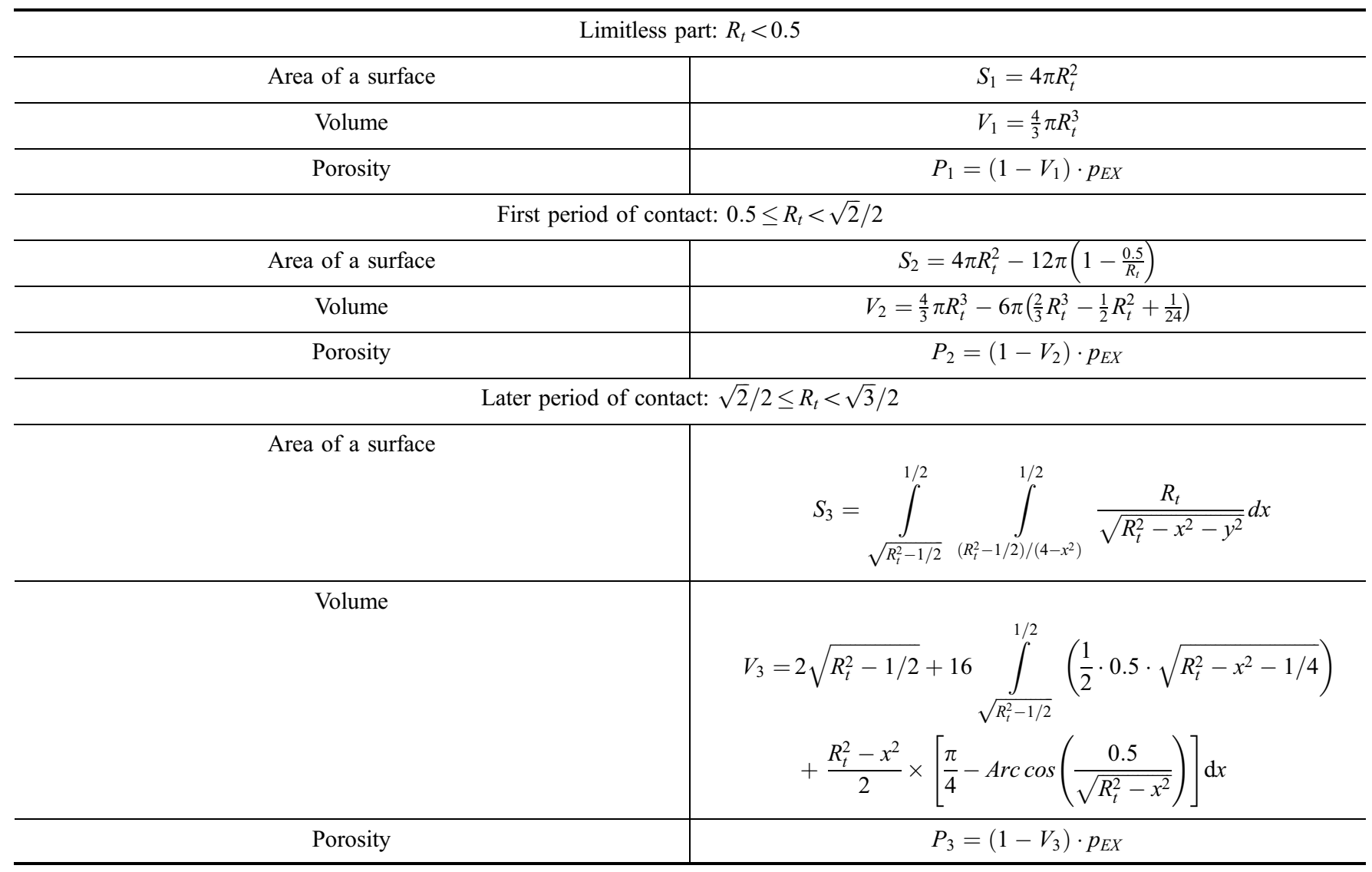

the compressive strength and elastic modulus show the same characteristic in relation to the porosity. However, the relationship between the porosity and compressive strength is expressed as a spatial probability distribution, which is dependent on the existence probability of the weakest point in the particle connections of the hardened cement, whereas the correlation between the porosity and elastic modulus is a parameter that represents the spatial structure that shows how the stress is transferred in the state before reaching the point of destruction (Maruyama 2003). Based on these characteristics, when the paste matrix is thought of as a hardened part of the gel, non-hydrated cement, and nonhydrated expansive additive, and as a two-phase pore material, elastic modulus models for the hardened part with and without pores are needed, both of which use the concept

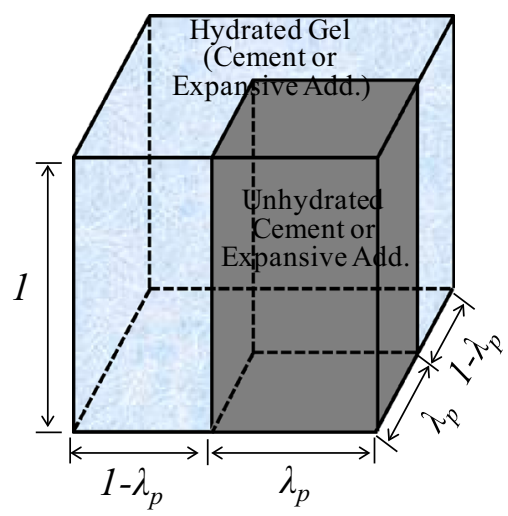

Fig. 6 Schematic view of elastic modulus of cement paste without pores. of the contact area. On the other hand, for a hardened cement mixed with the expansive additive, two elastic moduli are needed, one for the cement part and the other for the expansive additive part. In consideration of the mix ratio of the expansive additive, each elastic modulus must have a balancing equation.

In accordance with the proportion $\lambda_{P}$, which is occupied by the non-hydrated cement, non-hydrated expansive additive, and hydration product, the model of the elastic modulus for the nonporous part has the shape shown in Fig. 6. To model the spatial structure, the volume ratio $V_{C, E X}$ of the non-hydrated cement to non-hydrated expansive additive is needed, based on the following equation.

$$
\lambda_{P}=\sqrt{V_{C, E X}}
$$

On the other hand, in the modeling of the elastic modulus, we assume that the gel, non-hydrated cement, and nonhydrated expansive additive exist in a certain ratio based on the hydration reaction ratio.

$$
\begin{aligned}
& V_{C, E X}=\frac{(1-\alpha)}{(1-\alpha)+v_{g e l(C, E X)} \alpha} \\
& V_{g e l(C, E X)}=1-V_{C, E X}
\end{aligned}
$$

where $\alpha$ is the hydration reaction rate, $v_{\operatorname{gel}(C, E X)}$ is the volume ratio for the produced gel of the cement and expansive additive volumes that contribute to hydration, $V_{C, E X}$ is the volume ratio of the unhydrated cement or unhydrated expansive additive, and $V_{g e l(C, E X)}$ is the volume 
ratio of the gel. In this case, the elastic modulus can be expressed by the following equation.

$$
E_{u-p a s t e}(C, E X)=\frac{1}{\frac{\lambda_{p}}{\lambda_{p} \cdot E_{g e l(C, E X)}+\left(1-\lambda_{p}\right) \cdot E_{C, E X}}+\frac{1-\lambda_{p}}{E_{g e l(C, E X)}}}
$$

where $E_{u-p a s t e(C, E X)}$ is the elastic modulus of the hardened cement paste or hardened expansive paste without pores, $E_{C . E X}$ is the elastic modulus of the unhydrated cement or unhydrated expansive additive, and $E_{g e l(C . E X)}$ is the elastic modulus of the hydration product.

Here, $E_{C, E X}$ and $E_{g e l(C, E X)}$ have values of 50 and $25 \mathrm{GPa}$, respectively. These values were taken from the results of the study by Maruyama (2003) on the calculation of the elastic modulus of cement. Maruyama determined these values by referring to the values of 40 and $20 \mathrm{GPa}$ suggested by Hua et al. (1997) and 60 and $30 \mathrm{GPa}$ suggested by Lokhorst and van Breugel (1997). In the case of the expansive additive, the cement value was applied because sufficient data could not be found.

\subsection{Elastic Modulus of Cement Paste with Pore}

The elastic modulus with pores was represented in the CCBM model (Maruyama 2003) and modeled using the contact area concept, particularly the effective radius factor and effective contact area. The contact area in the C-CBM model is the characteristic value of the area that transfers stress through the connectivity of the particles. However, because the early hydration reaction occurs with the spatial expansion of an external product and becomes increasingly densified because of the hydration, it cannot be expressed using the contact area alone. That is, the early hydration reaction enlarges the radius of the external hydration product $R_{t}$, as shown in Fig. 7. Given that the thickness and density of the external product remain at a certain degree, the ratio of the actual external product to its thickness $R_{t}-r_{0}$ is the effective radius factor $E_{f r}$ and can be applied to the effective contact area $A_{\text {ceff }}$, which is used for modeling. This concept is also applied to the modeling of the elastic modulus with pores.

Effective radius factor $E_{f r}$ and effective contact area $A_{c e f f}$ of $\mathrm{C}-\mathrm{CBM}$ are shown in the following equations.
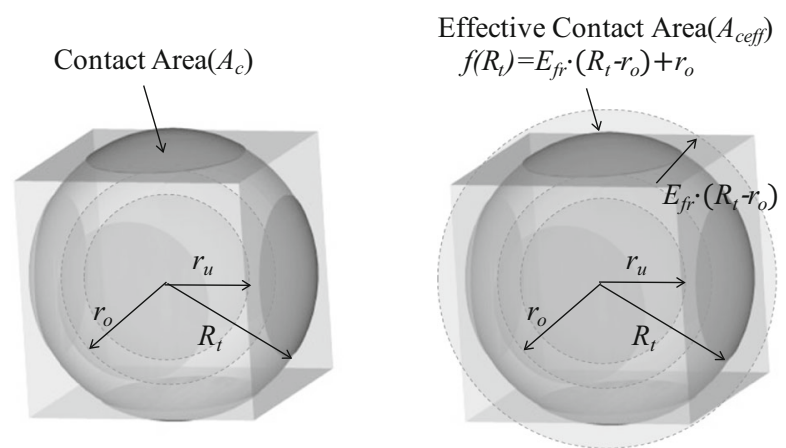

Fig. 7 Schematic view of effective radius factor and effective contact area.

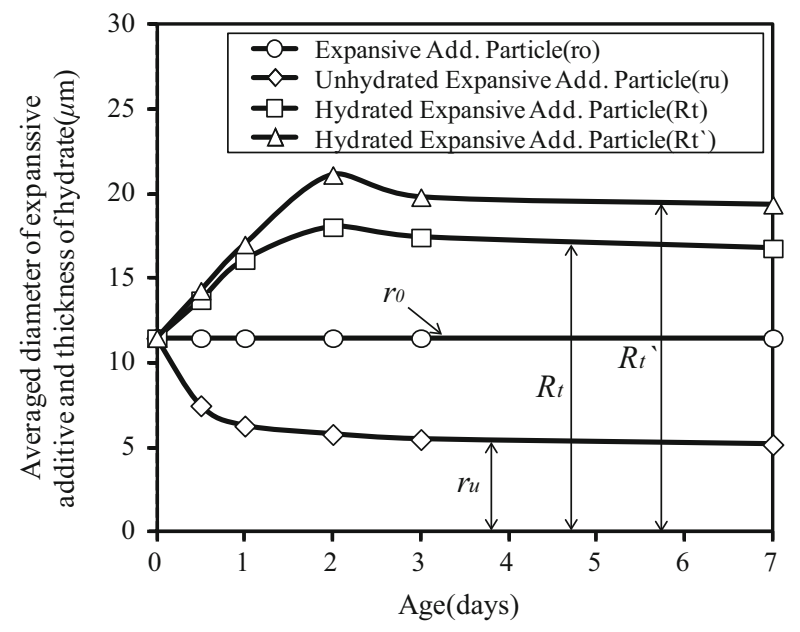

Fig. 8 Volume change of hydration layer of expansive additive by considering hydration at early age.

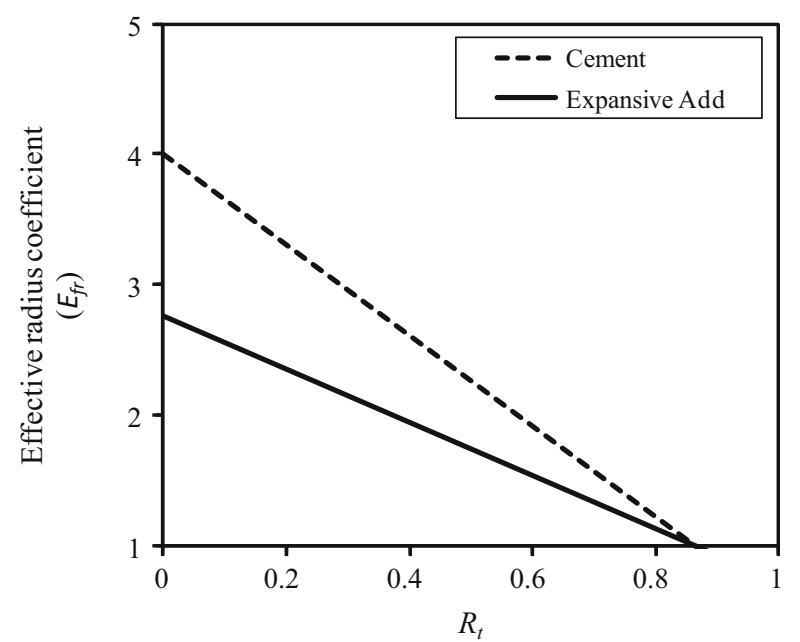

Fig. 9 Change in $\boldsymbol{E}_{f r}$ by $\boldsymbol{R}_{t}$.

$$
\begin{aligned}
& E_{f r}=E_{f r o} \cdot\left(1-\frac{2}{\sqrt{3}} R_{t}\right)+1 \\
& A_{\text {ceff }}=f\left(R_{t}\right), \quad f\left(R_{t}\right)=E_{f r} \cdot\left(R_{t}-r_{o}\right)+r_{o}
\end{aligned}
$$

where $E_{\text {fro }}$ is the initial value of the effective radius factor. In the case of cement, a value of 3.0 was applied, which was selected by giving equal consideration to the development of the elastic modulus in previous studies using the C-CBM model. In the case of the expansive additive, it was necessary to obtain the increase in the radius of external products by the hydration reaction of the expansive additive at an early age, as shown in Fig. 8, by referring to previous studies (Yanimoto et al. 2003). This was used to derive the effective radius factor of the expansive additive, which here has an initial value of 1.759. Figure 9 shows the change in the effective radius factor $E_{f r}$ by the outermost particle radius $R_{t}$ for the cement and expansive additive. The initial effective radius factors of the cement and expansive additive are different because the volume increase rate $V$ for the expansive additive already considers the pores generated as a result of the hydration reaction. Function $f\left(R_{t}\right)$ can be 
acquired using Eq. (3), which is a function to express the relation of the outermost particle radius $R_{t}$ and apparent effective contact area $A_{\text {ceff }}$.

Therefore, the elastic modulus of the cement paste part and expensive additive paste part $E_{\text {paste }(C, E X)}$ can be obtained by the following equation using the external contact area $A_{\text {ceff }}$.

$$
E_{\text {paste }(C, E X)}=E_{u-p a s t e(C, E X)} \cdot A_{c e f f}
$$

In addition, the elastic modulus of the paste mixed with the expansive additive can be expressed by the following equation, depending on the mixture rate of the expansive additive according to the balance between the required cement-part elastic modulus and expansive additive-part elastic modulus.

$$
E_{\text {paste }(C+E X)}=E_{\text {paste }(C)} \cdot C_{V(\%)}+E_{\text {paste }(E X)} \cdot E X_{V(\%)}
$$

where $E_{\text {paste }(C+E X)}$ is the elastic modulus of the cement paste mixed with the expansive additive, $E_{\text {paste }(C)}$ is the elastic modulus of the cement part, $E_{\text {paste(EX) }}$ is the elastic modulus of the expansive additive part, $C_{V(\%)}$ is the volume mixing rate of the cement, and $E X_{V(\%)}$ is the volume mixing rate of the expansive additive.

\subsection{Elastic Modulus of Concrete}

In order to extend the elastic modulus to concrete, the concrete is considered to consist of two materials, the aggregate and paste. It is also assumed that there is no behavior by the aggregate. Hence, the behavior of the paste dominates in the behavior of the concrete. Therefore, the aggregate has a resistor function without affecting the behavior of the paste.

Assuming that a composite model for the aggregate and paste is established based on the aforementioned assumptions and ideas, the elastic modulus of the concrete can be estimated from the elastic modulus of the paste, as shown in Fig. 10.

The elastic modulus of the concrete can be expressed by the following equation without considering Poisson's ratio.

$$
E_{\text {concrete }(C+E X)}=\frac{1}{\frac{\lambda_{c}}{\lambda_{c} \cdot E_{\text {paste }(C+E X)}+\left(1-\lambda_{c}\right) \cdot E_{a g g}}+\frac{1-\lambda_{c}}{E_{\text {paste }(C+E X)}}}
$$

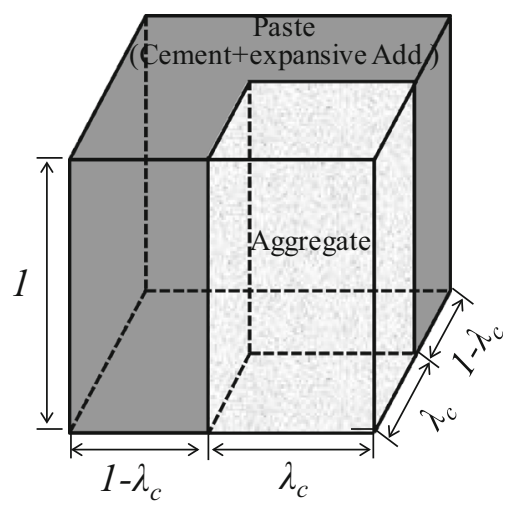

Fig. 10 Composite model of elastic modulus for concrete. where $E_{\text {concrete }(C+E X)}$ is the elastic modulus of the concrete mixed with the expansive additive, $E_{\text {paste }(C+E X)}$ is the elastic modulus of the cement paste mixed with the expansive additive, and $E_{\text {agg }}$ is the elastic modulus of the aggregate.

In addition, $\lambda_{c}$ is a constant of the volume of the aggregate $V_{\text {agg }}$ in the unit volume, and can be expressed by the following equation.

$$
\lambda_{c}=\sqrt{V_{a g g}}
$$

\section{Verification of Model}

\subsection{Compressive Strength}

An experiment was performed to verify the compressive strength model. Water and binder at a ratio of 0.50 was mixed with 0,5 , and $10 \%$ expansive additive (Ettringitegypsum type, Density $3.05 \mathrm{~g} / \mathrm{cm}^{3}$ ). The increase in the coefficient $p_{E X}$ of the capillary pores generated by the volume increase with this expansive additive admixture was set by referring to the studies of Yaniamoto and Morioka (Yanimoto et al. 2003; Morioka et al. 1999), in which a mercury porosimeter test measured the change in a specimen's porosity.

For the increase rate of the capillary pores following the mixing of the expansive additive, a regression equation was derived for the admixture of the expansive additive and the increase rate of the pores, as shown in Fig. 11. By considering this, the total porosity was found for the case of mixing in the expansive additive.

Figure 12 shows the change in the capillary pore volume of the paste. We confirmed that the capillary pore volume increased with the admixture of the expansive additive and decreased with the progress of the hydration. Figure 13 shows the compressive strength estimates found using the model and the values measured in an experiment, based on the total porosity. Because our water/binder ratio was 0.50 , the porosity found by the model was in the range of a high porosity of 0.3 or greater, and Eq. (5) from Schiller was used for the estimated values. The figure also shows Morioka's

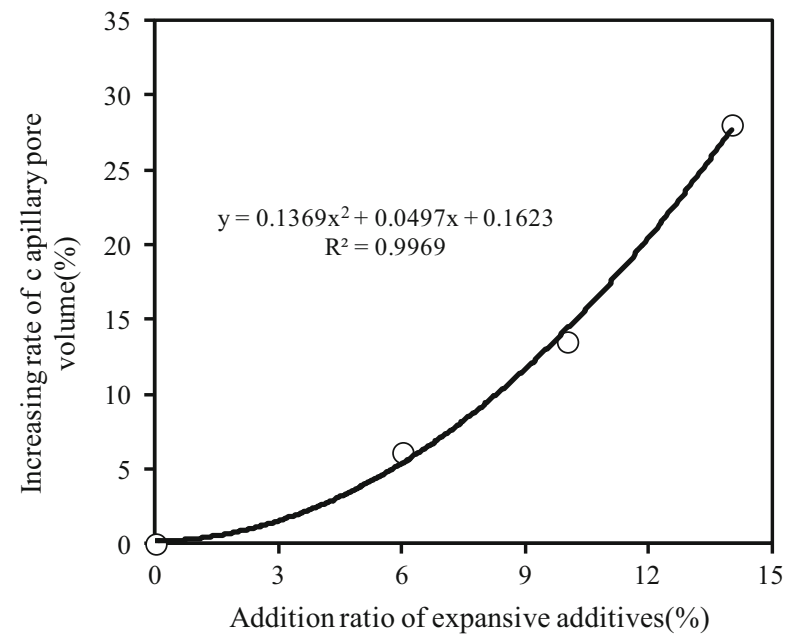

Fig. 11 Increasing rate of capillary pores by addition ratio of expansive additive. 


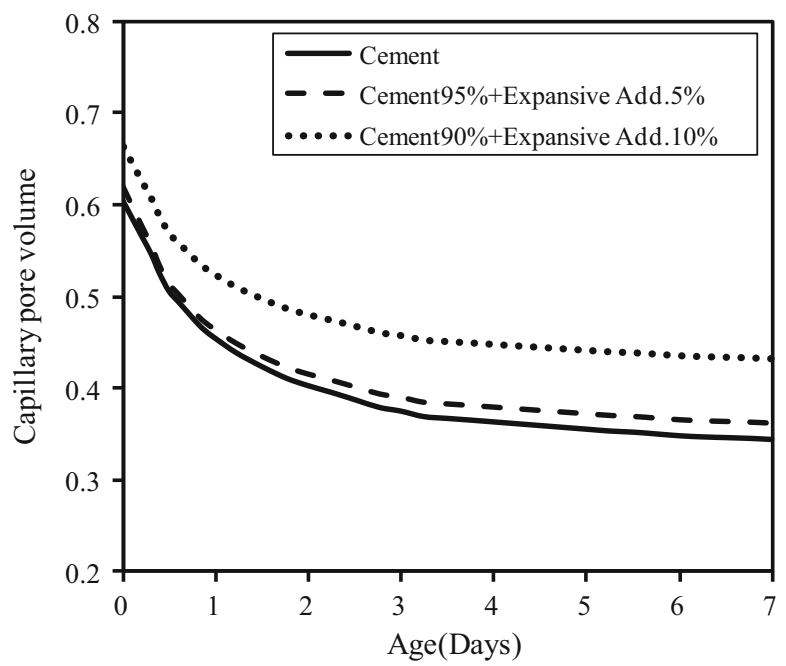

Fig. 12 Change in capillary pore volume.

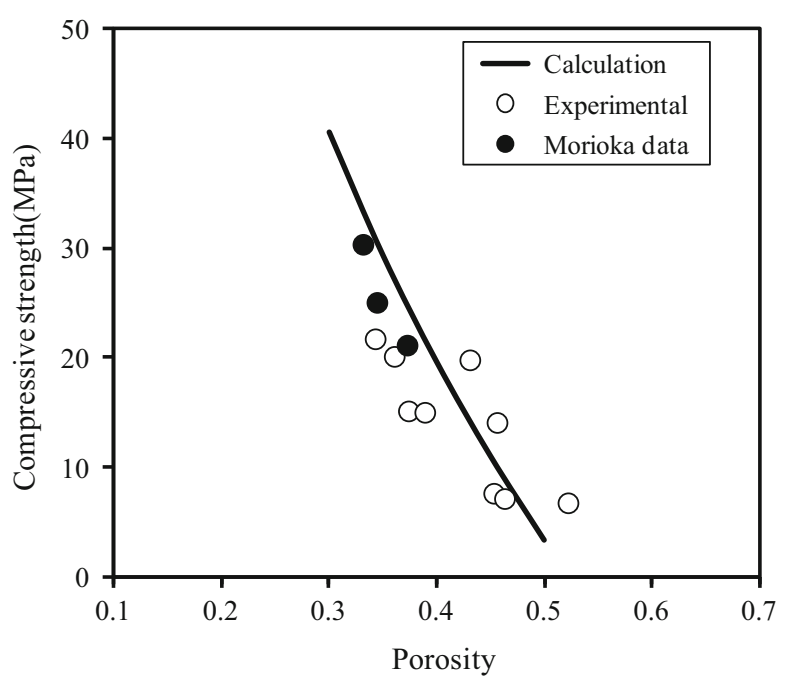

Fig. 13 Compressive strength development by model compared with experimental data.

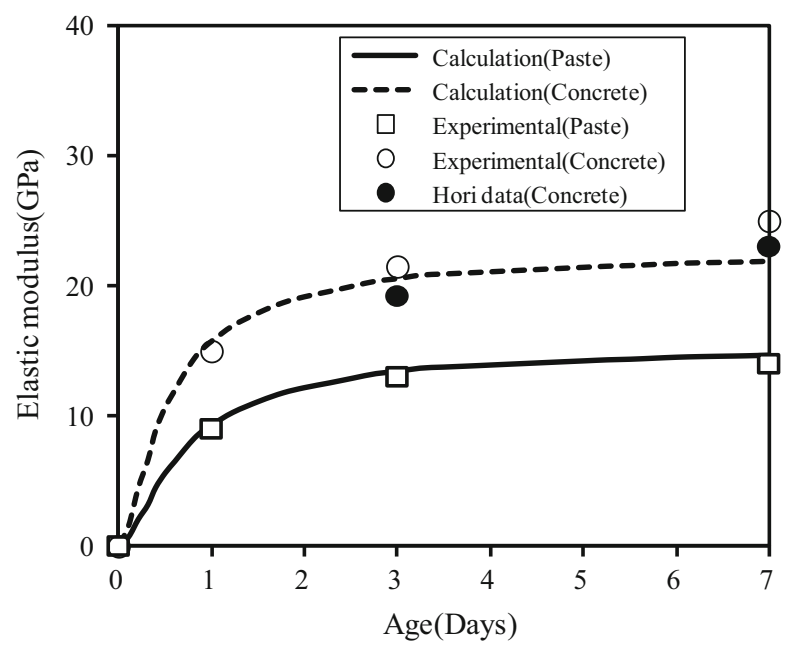

Fig. 14 Elastic modulus development by model compared with experimental data.

data (Morioka et al. 1999), in which the expansive additive was mixed at 10 and $12 \%$ and aged for 7 days, with a water/ binder ratio of 0.50 . As shown in the figure, we confirmed that the compressive strength estimates were positively correlated with the experiment values.

\subsection{Elastic Modulus}

To verify the elastic modulus model, we measured the elastic modulus of the cement paste after 1, 3, and 7 days, in which $10 \%$ expansive additive(Ettringite-gypsum type, Density $3.05 \mathrm{~g} / \mathrm{cm}^{3}$ ) was mixed as a binder, where the water/ binder ratio was 0.50 . The actual values for the paste measured in the experiment and the model's estimated values are shown in Fig. 14. In addition, this figure shows the estimated elastic modulus of the concrete and Hori's data for the elastic modulus of the concrete after 3 and 7 days, where the concrete included $30 \mathrm{~kg}$ (approximately $10 \%$ of the cement) of the expansive additive, with a water/binder ratio of 0.50 (Hori et al. 2000). Elastic modulus of aggregates were used $60 \mathrm{GPa}$ for the calculation on elastic modulus of concrete. As shown in the figure, we confirmed that the elastic modulus estimates were positively correlated with the experiment values. It was confirmed that the aging of the elastic modulus of the concrete followed the estimate suggested in Hori's data and experimental data, indicating that the composite model can estimate the elastic modulus of concrete based on the behavior of the paste.

\section{Conclusions}

In this study, the compressive strength and elastic modulus of a hardened cement paste mixed with an expansive additive to control cracking were modeled, and the models were verified. The results of the study are summarized below.

(1) In the modeling of the mechanical properties of the concrete using the expansive additive, in the hardened cement paste with an expansive additive, hydrates are generated as a result of the hydration between the cement and expansive additive; these hydrates then fill up the pores in the hardened cement. Consequently, a dense, compact structure is formed through the contact between the particles of the expansive additive and the cement, leading to the manifestation of the strength and elastic modulus. Hence, modeling was performed to evaluate the compressive strength by assuming that the strength development of the cement paste was closely related to the pore volume.

(2) On the other hand, the elastic modulus of the hardened part without pores was modeled by assuming that the paste matrix consisted of the gel, non-hydrated cement, and hardened part of the non-hydrated expansive additive, with two-phase pores in the case where it contained pores. The elastic modulus was modeled using the concept of the effective radius factor and effective contact area.

(3) The estimates of the models were positively correlated with experimental values, which verified the compressive strength model and elastic modulus model. In addition, the elastic modulus of the concrete could 
effectively be estimated based on the composite model of the aggregate and paste.

\section{Open Access}

This article is distributed under the terms of the Creative Commons Attribution 4.0 International License (http://creativecommons.org/licenses/by/4.0/), which permits unrestricted use, distribution, and reproduction in any medium, provided you give appropriate credit to the original author(s) and the source, provide a link to the Creative Commons license, and indicate if changes were made.

\section{References}

Al-Rawi, R. S. (1976). Choice of curing temperature for accelerated cured concrete. Cement and Concrete Research, 16(5), 603-611.

Choi, H. G., Tsujino, M., Kitagaki, R. \& Noguchi, T. (2012a). Expansion-contraction behaviors and cracking control effects of expansion concrete in buildings. In Proceedings of the 5th ACF international conference, Pattaya, Thailand. [ACF2012-0096 (Session 3-2)].

Choi, H. G., Tsujino, M., Noguchi, T., \& Kitagaki, R. (2012b). Expansion/contraction behavior and cracking control effect of expansive concrete in building structure. Proceedings of the Japan Concrete Institute, 34(1), 424-429.

Hori, A., Tamaki, T., \& Hagiwara, H. (2000). Cracking behavior of expansive concrete in homoaxial tension. Proceedings of the Japan Concrete Institute, 22(2), 511-516.

Hua, C., Ehrlacher, A., \& Acker, P. (1997). Analyses and models of the autogenous shrinkage of hardening cement paste II modeling at scale of hydration grains. Cement and Concrete Research, 27(2), 245-258.

Katsura, O., Morimoto, J., \& Nawa, T. (1996). A model of strength development considering hydration of cement. Proceedings of the Japan Concrete Institute, 39, 109-114.

Lokhorst, S. J., \& van Breugel, K. (1997). Simulation of the effect of geometrical changes of the microstructure on the deformational behavior of hardening concrete. Cement and Concrete Research, 27(10), 1465-1479.

Maruyama, I. (2003). Time dependent property of cement based materials on the basis of micro-mechanics. PhD Dissertation, The University of Tokyo, Tokyo, Japan.

Morioka, M., Hagiwara, H., Sakai, E., \& Daimon, M. (1999). Chemical shrinkage and autogenous volume change of cement paste with expansive additive. Proceedings of the Japan Concrete Institute, 21(2), 157-162.

Park, S. G. (2004). A study on the reduction of autogenous shrinkage of high performance concrete using shrinkage reducing admixture and expansive additive at early age. PhD Dissertation, The University of Tokyo, Tokyo, Japan.

Park, K. B., \& Lee, H. S. (2005). Prediction of temperature profile in high strength concrete structures using a hydration model. Journal of the Architectural Institute of Korea Structure \& Construction, 21(10), 111-118.

Powers, T. C., \& Brownyard, T. L. (1947). Studies of the physical properties of hardened Portland cement paste. Journal of American Concrete Institute (Proceedings), 43.

Ryshkewitch, E. (1953). Composition and strength of porous sintered alumina and zirconia. Journal of the American Ceramic Society, 36, 65-68.

Schiller, K. K. (1958). Mechanical properties of non-metallic materials (pp. 35-50). London, UK: Butterworths.

Taplin, J. H. (1959). A method for following the hydration reaction in Portland cement paste. Australian Journal of Applied Sciences, 10, 329-345.

Tashiro, C. (1993). Water behavior in cement \& concrete. Report of water subcommittee, research committee of cement \& concrete.

van Breugel, K. (1997). Simulation of hydration and formation of structure in hardening cement-based materials. $\mathrm{PhD}$ Dissertation, Delft University, Delft, Netherlands.

Woods, H. (1933). Heat evoloved by cement in relation to strength. Engineering News-Record, 1933, 431-433.

Yanimoto, K., Morioka, M., Sakai, E., \& Daimon, M. (2003). Expansion mechanism of cement added with expansive additive. Concrete research and technology, Japan Concrete Institute, 14(3), 23-31. 